\title{
Flexible Contact Pressure Sensor Based on Ultrathin Piezoresistive Silicon Membrane Capable of Strain Compensation
}

\author{
Yusuke Takei, ${ }^{1 *}$ Shintaro Goto, ${ }^{2}$ Seiichi Takamatsu, ${ }^{2}$ \\ Toshihiro Itoh, ${ }^{2}$ and Takeshi Kobayashi ${ }^{1}$ \\ ${ }^{1}$ Research Center for Ubiquitous MEMS and Micro Engineering (UMEMSME), National Institute of Advanced \\ Industrial Science and Technology (AIST), 1-2-1 Namiki, Tsukuba, Ibaraki 305-8564, Japan \\ ${ }^{2}$ Graduate School of Frontier Sciences, The University of Tokyo, \\ 5-1-5 Kashiwanoha, Kashiwa-shi, Chiba 277-8563, Japan
}

(Received June 26, 2018; accepted October 22, 2018)

Keywords: pressure sensor, strain sensor, ultrathin silicon, piezoresistive silicon membrane, flexible device

In this study, we developed a highly sensitive and flexible contact pressure sensor by transferring an ultrathin piezoresistive silicon membrane to a flexible substrate. The sensor consists of two sensors, a contact pressure sensor and a strain sensor for strain compensation. From the output of these two sensors, strain caused by fixing the sensor to the curved surface can be compensated for, so that we can accurately measure contact pressure. In the experiments, we confirmed that the fabricated contact pressure sensor has the same sensitivity on both a flat surface and a curved surface. We also confirmed that our sensor can correctly measure contact pressure by compensating for the distortion when fixed to a curved surface.

\section{Introduction}

In recent years, MEMS sensors have been utilized in various situations. Since the MEMS sensors are small and lightweight, there is an increasing need to fix them not only on hard and flat surfaces but also on soft and curved surfaces. Therefore, research for realizing a sensor element and wiring with a flexible shape is actively conducted. ${ }^{(1-16)}$ Particularly for a flexible force sensor and a strain sensor element, it is common to make use of the piezoresistivity of conductive ink or a conductive polymer material. ${ }^{(17-44)}$ Although these materials are flexible, they have a gauge factor of about 1 to 5 , which is insufficient compared with the piezoresistive silicon strain gauge factor of 100 .

In general, however, a sensor based on piezoresistive silicon has a thickness of $500 \mu \mathrm{m}$ or more, and there is the problem that such a sensor breaks when bent. The reason why the silicon wafer is $500 \mu \mathrm{m}$ or thicker is that in the MEMS process, the flatness of the wafer is important to enable uniform processing. Such a thickness is also necessary to prevent the wafer from being deflected. For these reasons, it is difficult to realize a flexible pressure sensor using highly sensitive piezoresistive silicon. On the other hand, thin silicon having a thickness of $50 \mu \mathrm{m}$ or *Corresponding author: e-mail: yusuke-takei@aist.go.jp https://doi.org/10.18494/SAM.2018.2033 
less does not break even if it is bent. Therefore, in recent years, research has been conducted to realize a flexible semiconductive silicon device by transferring a thin MEMS device onto a flexible substrate; this is called flexible hybrid electronics (FHE). ${ }^{(45-51)}$ In our research group, Yamashita and co-workers developed a flexible strain sensor array sheet by transferring a thin lead zirconate titanate (PZT) film with a thickness of $10 \mu \mathrm{m}$ fabricated on a silicon-on-insulator (SOI) wafer onto a flexible substrate. ${ }^{(52-56)}$ However, although the PZT sensor is suitable for dynamic distortion measurement, it was not suitable for measuring static distortion or distortion caused by low-frequency force such as contact pressure. Therefore, in this research, we used an ultrathin piezoresistive silicon membrane to realize a flexible sensor that can measure static strain and contact pressure.

Generally, when piezoresistive silicon is distorted, the resistance changes. Therefore, when a flexible sensor with piezoresistive silicon is fixed to a curved surface, the resistance changes owing to distortion. Since this resistance change continues to be added to the sensor output, there is the problem that it cannot be separated from the resistance change due to the strain caused by the contact pressure. In this research, we fabricated a "contact pressure sensor" and a "strain sensor" side by side in a flexible structure [Fig. 1(1)]. From the output of these two sensors, strain caused by fixing the sensor to the curved surface can be compensated, so that we can accurately measure contact pressure even if the sensor is attached to a curved surface of arbitrary shape [Fig. 1(2)].

(1)

Fixed on flat surface

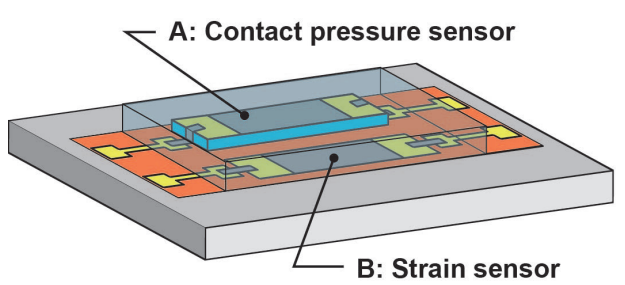

(2)

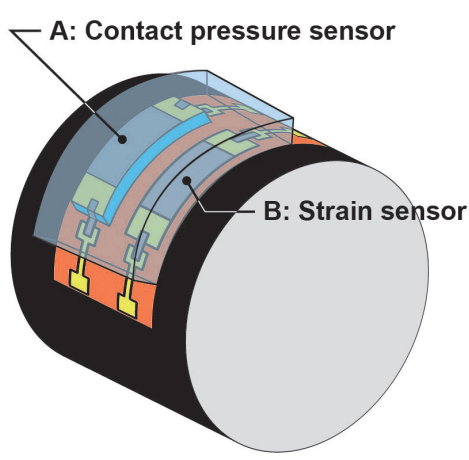

Fig. 1. (Color online) Measurement principle of our proposed sensor. 


\section{Proposed Sensor}

The principle of detecting the contact pressure while compensating for the strain when the proposed sensor is fixed to a curved surface is described as follows. As shown in Fig. 1(1), the sensor consists of two sensors, A: contact pressure sensor and B: strain sensor. In the contact pressure sensor, a 5 - $\mu \mathrm{m}$-thick piezoresistive silicon membrane is mounted on a 0.3 -mm-thick silicone rubber layer formed on a polyimide substrate, and a $0.7-\mathrm{mm}$-thick silicone rubber layer covers the membrane [Fig. 1(3) A-(a)]. On the other hand, the strain sensor is composed of a 5 - $\mu \mathrm{m}$-thick piezoresistive silicon membrane fixed on a polyimide substrate and covered with 1-mm-thick silicone rubber [Fig. 1(3) B-(a)].

When the strain sensor and the contact pressure sensor are formed close to each other, as shown in Fig. 1(1), and fixed to a curved solid surface, as shown in Fig. 1(2), both piezoresistive silicon membranes are distorted by the same amount, which causes the resistance change $\Delta R_{\varepsilon}$ [Figs. 1(3) A-(b), B-(b)]. When contact pressure is applied to the sensor fixed to the curved surface, as shown in Figs. 1(3) A-(c), B-(c), the strain sensor is not deformed by the contact pressure, and additional resistance change does not occur. On the other hand, in the case of the contact pressure sensor, the ultrathin piezoresistive silicon membrane will be distorted by the applied contact pressure and resistance change $\Delta R_{p}$ will occur as a result of the extra deformation. Therefore, by taking the difference between the resistance changes of these two sensors, we can measure the resistance change $\Delta R_{p}$ due to contact pressure.

\section{Fabrication}

In this section, we describe the fabrication process for the proposed flexible contact pressure sensor. Figure 2 shows the overall process flow. This process is roughly divided into two: the process of fabricating an ultrathin piezoresistive silicon membrane from an SOI wafer, and the process of transferring the ultrathin piezoresistive silicon membrane to a flexible substrate and coating it with silicone rubber (KE-108, Shin-Etsu Silicone, Japan).

First, we fabricated the $5-\mu \mathrm{m}$-thick ultrathin piezoresistive silicon membrane of the device layer on the $5 / 2 / 500 \mu \mathrm{m}$ SOI wafer. To form a piezoresistive layer on the surface of the devicelayer silicon (P type), a phosphorus ion dopant (OCD P-59230, Tokyo Ohka Kogyo Co., Ltd, Japan) was spin-coated, and thermal diffusion was performed in an annealing furnace. After that, an electrode was formed, and the device-layer silicon was etched by deep reactive-ion etching (DRIE) to form the ultrathin piezoresistive silicon membrane [Fig. 2(1)]. The membrane has a rectangular shape of $1 \times 5 \mathrm{~mm}^{2}$. Then, the handling-layer silicon was patterned and etched from the back, so that a 5 - $\mu$ m-thick ultrathin piezoresistive silicon membrane supported by four beams was fabricated, as shown in Fig. 2(2).

Next, the process of transferring the fabricated silicon membrane onto a flexible substrate and coating with silicone rubber will be described. Figures 2(3) to 2(7) show the fabrication process of the flexible contact pressure sensor. The proposed flexible pressure sensor consists of two types of sensors: a flexible pressure sensor for pressure measurement and a flexible strain sensor for strain compensation. The difference in the manufacturing processes of these 


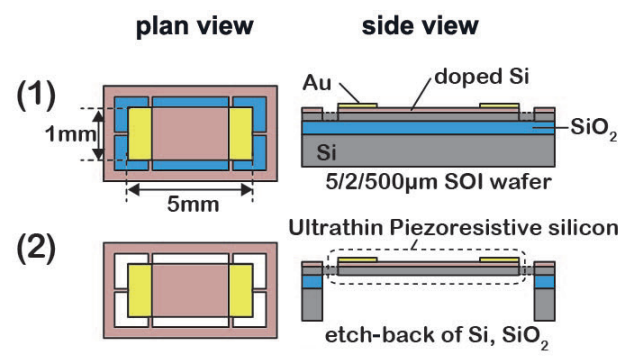

(3)

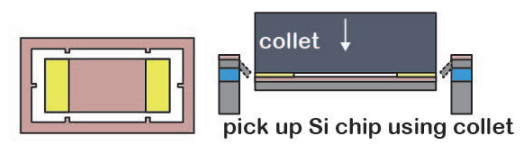

Contact Pressure sensor

Strain sensor

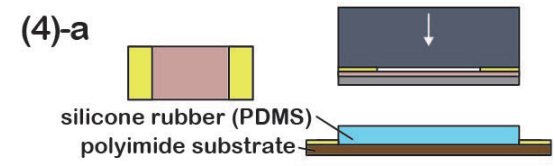

(5)-a

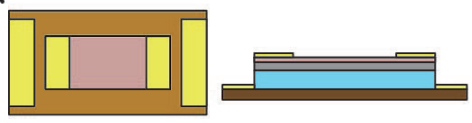

(6)-a

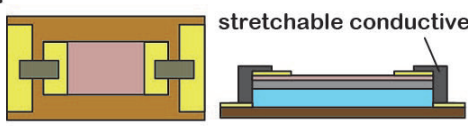

(7)-a

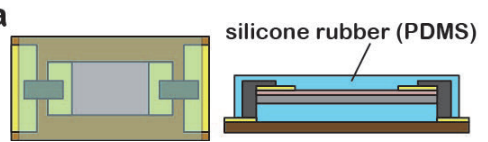

(4)-b

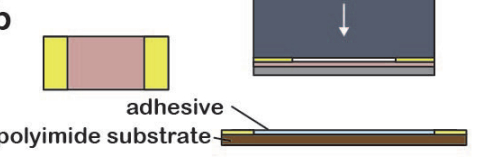

(5)-b

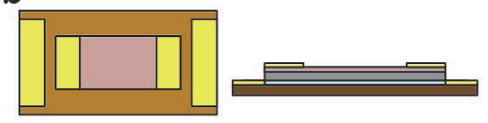

(6)-b

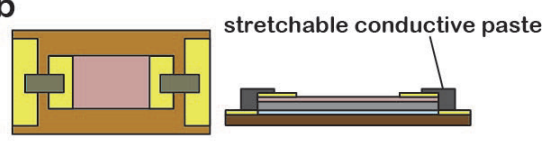

(7)-b

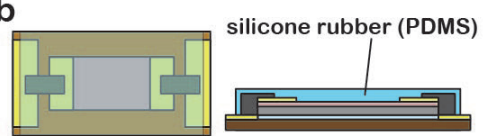

Fig. 2. (Color online) Fabrication process.

two sensors is that the strain sensor is placed directly on the polyimide substrate [Figs. 2(4)-b to 2(7)-b)], whereas the contact pressure sensor is placed on a silicone rubber layer with a thickness of $300 \mu \mathrm{m}$ [Figs. 2(4)-a to 2(7)-a]. In this research, we selected polyimide as the base material for the flexible sensor, as metal wiring can be easily patterned and it can withstand the thermal curing temperature $\left(80^{\circ} \mathrm{C}\right)$ of polydimethylsiloxane (PDMS). For membrane transfer, we use a flip chip bonder (M-400, HiSOL, Inc., USA). As shown in Fig. 2(3), by slightly pressing the collet against the membrane, the four supporting beams are broken and the membrane is lifted up by vacuum suction. Thereafter, this membrane is aligned and pressed onto the polyimide substrate. After the transfer, the copper electrode on the polyimide substrate and the gold electrode of the piezoresistive silicon membrane are connected with stretchable conductive paste. Then, silicone rubber is poured onto the surface and cured to adjust the total thickness to $700 \mu \mathrm{m}$. Figure 3 shows the transferred ultrathin piezoresistive silicon membrane. Flexible pressure sensors and strain sensors are transferred nearby. Also, since the sensor is thin, about 0.7 $\mathrm{mm}$ thick, it is composed only of a flexible material, so that breakage and disconnection of the wiring and the sensor membrane do not occur even if it is bent between the fingers, as shown in Fig. 4. 

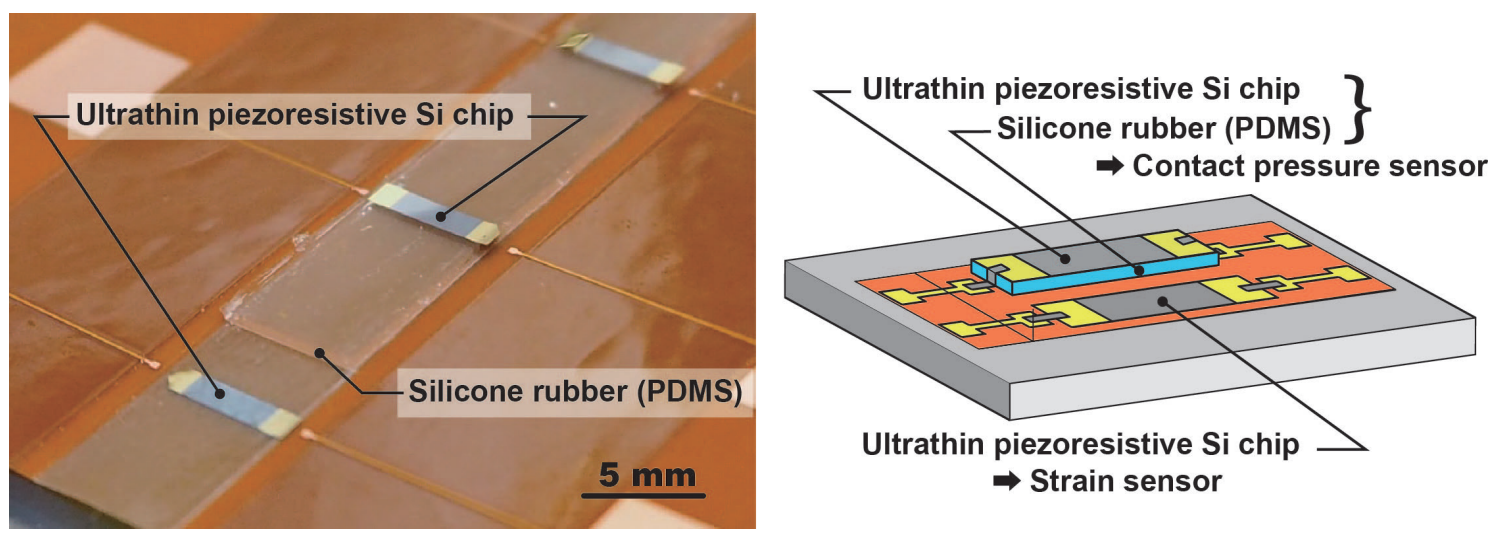

Fig. 3. (Color online) Photograph of transferred ultrathin piezoresistive silicon membranes on polyimide substrate and silicone rubber.

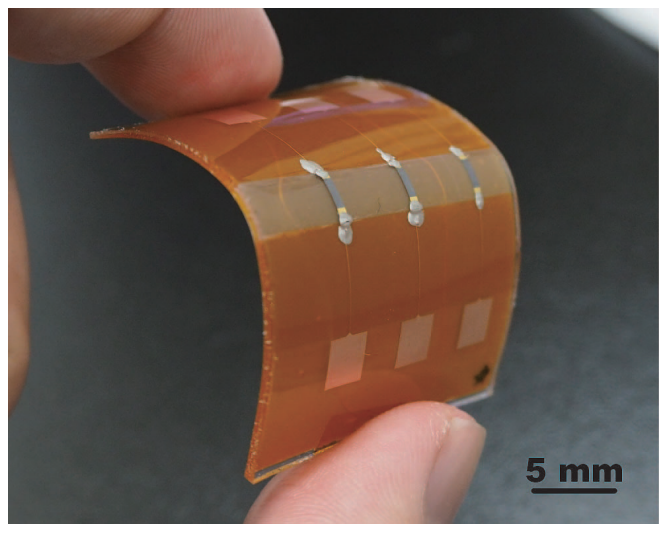

Fig. 4. (Color online) Photograph of the fabricated sensors.

\section{Results and Discussion}

To evaluate the fabricated flexible contact pressure sensor, the following three experiments were conducted: (I) piezoresistivity evaluation of fabricated ultrathin piezoresistive silicon membrane transferred onto the polyimide substrate; (II) response of the sensor to the contact pressure when the sensor is attached to a flat and a curved surface; and (III) measurement of the contact pressure with strain compensation when the sensor is fixed on a curved surface.

\subsection{Piezoresistivity evaluation}

First, the relationship between strain and resistance change of the fabricated ultrathin piezoresistive silicon membrane was evaluated. The polyimide substrate onto which the piezoresistive silicon membrane was transferred was pulled in the elongation direction, and the strain and resistance change at that time were measured. The results are shown in Fig. 5. We confirmed that the gauge factor of the fabricated piezoresistive silicon membrane was about 100 . 


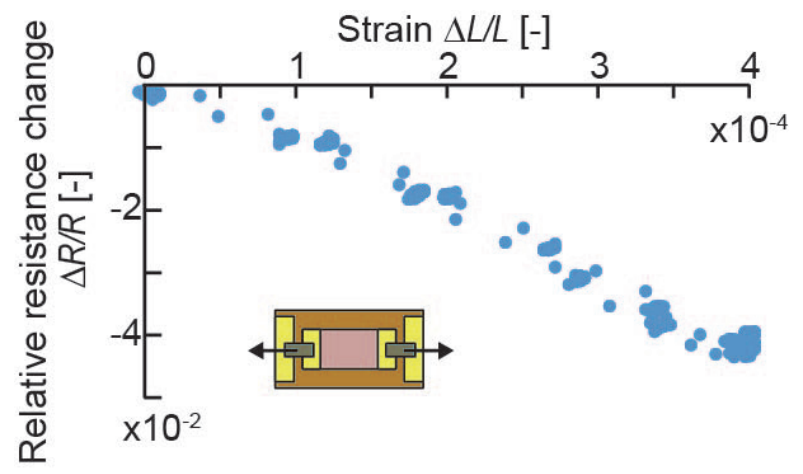

Fig. 5. (Color online) Relationship between strain and resistance change of fabricated ultrathin piezoresistive silicon membrane.

\subsection{Response of sensor to contact pressure}

To confirm whether the fabricated flexible pressure sensor can measure the contact pressure on both a flat surface and a curved surface, the resistance change with respect to the contact pressure when the sensor is fixed on a flat surface and on a curved surface was measured. In this experiment, the sensor was fixed on the surface of an acrylic pipe with a diameter of $5.5 \mathrm{~cm}$ as the curved surface, and contact pressure was applied to the sensor using a circular jig with a diameter of $10 \mathrm{~mm}$ so that both pressure and strain sensors can be simultaneously covered and pressure can be applied. Figure 6 shows the results. From the measurement result, it was confirmed that the sensors fixed to the plane and to the curved surface both exhibit a resistance change ratio in linear proportion to the applied contact pressure. Furthermore, from the slope of the linear relationship on the graph, it was confirmed that the same sensitivity to pressure is demonstrated for both flat and curved surfaces. This indicates that the contact pressure applied to the sensor can be correctly measured even when it is fixed to a curved surface, owing to the strain compensation based on the response of the strain sensor fabricated near the contact pressure sensor.

\subsection{Measurement of contact pressure with strain compensation}

Finally, experiments were conducted to confirm that the contact pressure can be correctly measured by compensating for the strain even when the fabricated flexible pressure sensor is fixed to a curved surface. Figure 7 shows the measurement results for a sensor attached to an acrylic pipe with a diameter of $5.5 \mathrm{~cm}$ and touched with a finger. The measured results shown in Fig. 7 are divided into three stages. (i) First, before attaching the sensor to the curved surface, the sensor was lightly bent three times by hand. (ii) Then, the sensor was fixed on the curved surface, and (iii) pressure was applied by touching the sensor surface three times with a finger. The three graphs in Fig. 7 show the outputs of the contact pressure sensor, the strain sensor, and the strain-compensated sensor. 


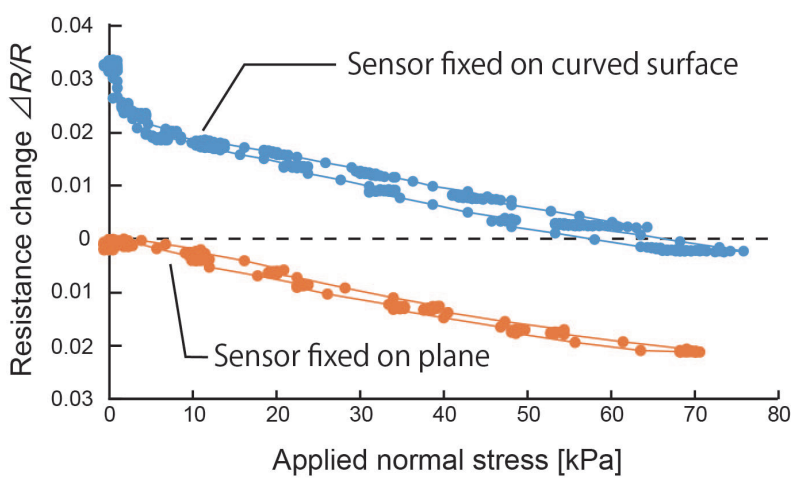

Fig. 6. (Color online) Relationship between applied contact pressure and resistance change for sensors fixed on a plane and a curved surface.

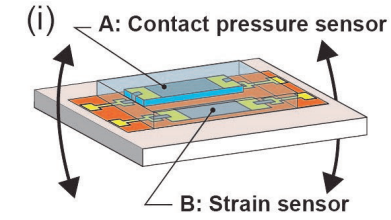

(ii) - A: Contact pressure sensor

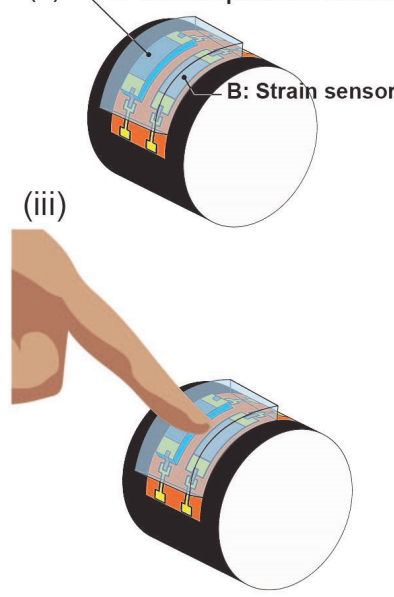

(i)

(ii)

(iii)

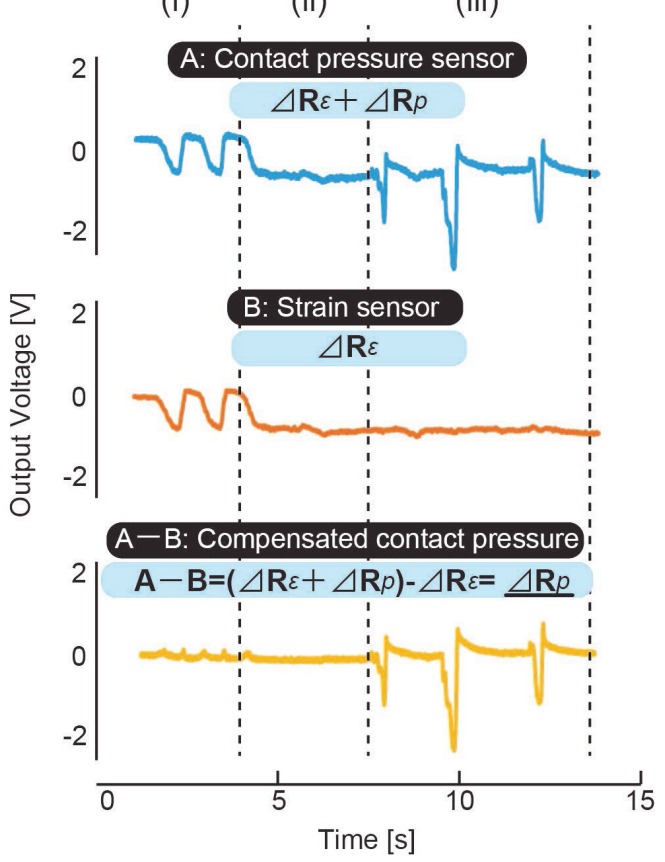

Fig. 7. (Color online) Results of measuring contact pressure with strain compensation.

In stage (i), it is confirmed that strain due to bending occurs in both the contact pressure sensor and the strain sensor. From the difference between these two sensor outputs, we confirmed that the strain due to bending is compensated and the sensor output is canceled to zero. In stage (ii), by fixing the sensor onto a curved surface, the same amount of strain was generated in the contact pressure sensor and the strain sensor, and it was also confirmed that the sensor output was canceled to 0 as a result of compensating for the strain. In stage (iii), when the sensor fixed on the curved surface was touched with a finger, only the contact pressure sensor responded, because of the structure of the sensor where the contact pressure sensor has a silicone rubber layer under the ultrathin piezoresistive silicon membrane. In this experiment, 
there was a PDMS layer between the piezoresistive Si membrane and the finger, and the contact time was less than $0.5 \mathrm{~s}$, so apparently, there was almost no influence of the body temperature. When the sensor was pushed for a longer contact time, resistance changed owing to temperature change in both the pressure sensor and the strain sensor. However, it is considered that by taking the difference between the two sensors, the resistance change due to the temperature change will be canceled. As a result, it was confirmed that the bending and distortion arising when the sensor was fixed to a curved surface were compensated and only the response to contact pressure was measured.

\section{Conclusions}

In this study, we developed a highly sensitive and flexible contact pressure sensor by transferring an ultrathin piezoresistive silicon membrane to a flexible substrate. The sensor consists of two sensors, a contact pressure sensor and a strain sensor for strain compensation. We fabricated these two sensors side by side in a flexible structure. From the output of these two sensors, we found that the strain caused by fixing the sensor to the curved surface can be compensated, so that contact pressure can be accurately measured even if the sensor is attached to a curved surface of arbitrary shape. From the experimental results, we confirmed that the fabricated contact pressure sensor had the same sensitivity on both a flat surface and a curved surface. We also confirmed that our sensor can correctly measure contact pressure because the distortion caused by attachment to a curved surface is compensated.

\section{References}

1 M. Cuartero, J. S. del Rio, P. Blondeau, J. A. Ortuno, F. X. Rius, and F. J. Andrade: Anal. Chim. Acta 827 (2014) 95.

2 S. Cruz, L. A. Rocha, and J. C. Viana: J. Mater. Sci. Mater. Electron. 28 (2017) 2563.

3 H. Jin, N. Matsuhisa, S. Lee, M. Abbas, T. Yokota, and T. Someya: Adv. Mater. 29 (2017) 1605848.

4 M. Abas and K. Rahman: Appl. Phys. A 122 (2016) 972.

5 N. Matsuhisa, M. Kaltenbrunner, T. Yokota, H. Jinno, K. Kuribara, T. Sekitani, and T. Someya: Nat. Commun. 6 (2015) 7461.

6 K. Tian, J. Bae, S. E. Bakarich, C. H. Yang, R. D. Gately, G. M. Spinks, M. I. H. Panhuis, Z. G. Suo, and J. J. Vlassak: Adv. Mater. 29 (2017) 1604827.

7 M. Santhiago, C. C. Correa, J. S. Bernardes, M. P. Pereira, L. J. M. Oliveira, M. Strauss, and C. C. B. Bufon: ACS Appl. Mater. Interfaces 9 (2017) 24365.

8 G. I. Hay, D. J. Southee, P. S. A. Evans, D. J. Harrison, G. Simpson, and B. J. Ramsey: Sens. Actuators, A 135 (2007) 534.

9 N. Karim, S. Afroj, A. Malandraki, S. Butterworth, C. Beach, M. Rigout, K. S. Novoselov, A. J. Casson, and S. G. Yeates: J. Mater. Chem. C 5 (2017) 11640.

10 Z. D. Li, H. Liu, C. Ouyang, W. H. Wee, X. Y. Cui, T. J. Lu, B. Pingguan-Murphy, F. Li, and F. Xu: Adv. Funct. Mater. 26 (2016) 165.

11 M. Borghetti, M. Serpelloni, E. Sardini, and S. Pandini: Sens. Actuators, A 243 (2016) 71.

12 S. Bai, S. G. Zhang, W. P. Zhou, D. L. Ma, Y. Ma, P. Joshi, and A. M. Hu: Nano-Micro Lett. 9 (2017) 42.

13 N. N. Jason, M. D. Ho, and W. L. Cheng: J. Mater. Chem. C 5 (2017) 5845.

14 V. Sencadas, R. Mutiu, and G. Alici: Sens. Actuators, A 266 (2017) 56.

15 S. Agarwala, G. L. Goh, Y. L. Yap, G. D. Goh, H. Yu, W. Y. Yeong, and T. Tran: Sens. Actuators, A 263 (2017) 593.

16 J. Ko, S. Jee, J. H. Lee, and S. H. Kim: Sens. Actuators, A 274 (2018) 50.

17 X. L. Zhao, Q. L. Hua, R. M. Yu, Y. Zhang, and C. F. Pan: Adv. Electron. Mater. 1 (2015) 1500142. 
18 X. X. Zhang, S. T. Hu, M. Wang, J. Yu, Q. Khan, J. T. Shang, and L. Ba: Nanotechnology 26 (2015) 115501.

19 D. B. Zhou and H. P. Wang: Sens. Actuators, A 204 (2013) 114.

20 D. Janczak, M. Sloma, G. Wroblewski, A. Mlozniak, and M. Jakubowska: Sensors 14 (2014) 17304.

21 M. Ha, S. Lim, J. Park, D. S. Um, Y. Lee, and H. Ko: Adv. Funct. Mater. 25 (2015) 2841.

22 B. Q. Nie, R. Y. Li, J. D. Brandt, and T. R. Pan: Lab Chip 14 (2014) 1107.

23 S. Chun, Y. Kim, H. Jung, and W. Park: Appl. Phys. Lett. 105 (2014) 041907.

24 S. Yoon, J. K. Sim, and Y. H. Cho: Sci. Rep. 6 (2016) 23468.

25 B. H. Liang, W. J. Chen, Z. F. He, R. L. Yang, Z. Q. Lin, H. W. Du, Y. Y. Shang, A. Y. Cao, Z. K. Tang, and X. C. Gui: Small 13 (2017) 1702422.

26 T. E. A. de Oliveira, A. M. Cretu, and E. M. Petriu: IEEE Sens. J. 17 (2017) 3231.

27 J. Wang, J. T. Jiu, T. Araki, M. Nogi, T. Sugahara, S. Nagao, H. Koga, P. He, and K. Suganuma: Nano-Micro Lett. 7 (2015) 51.

28 B. Q. Nie, R. Y. Li, J. Cao, J. D. Brandt, and T. R. Pan: Adv. Mater. 27 (2015) 6055.

29 L. Z. Sheng, Y. Liang, L. L. Jiang, Q. Wang, T. Wei, L. T. Qu, and Z. J. Fan: Adv. Funct. Mater. 25 (2015) 6545 .

30 S. Khan, S. Tinku, L. Lorenzelli, and R. S. Dahiya: IEEE Sens. J. 15 (2015) 3146.

31 S. C. B. Mannsfeld, B. C. K. Tee, R. M. Stoltenberg, C. V. H. H. Chen, S. Barman, B. V. O. Muir, A. N. Sokolov, C. Reese, and Z. N. Bao: Nat. Mater. 9 (2010) 859.

32 Z. P. Ji, H. Zhu, H. C. Liu, N. Liu, T. Chen, Z. Yang, and L. N. Sun: Sensors 16 (2016) 2001.

33 E. Choi, O. Sul, and S. B. Lee: Sensors 17 (2017) 2040.

34 J. C. Costa, A. Wishahi, A. Pouryazdan, M. Nock, and N. Munzenrieder: Adv. Electron. Mater. 4 (2018) 1700600.

35 F. G. Carrasco, D. D. Alonso, and L. Nino-de-Rivera: Microelectron. Eng. 159 (2016) 32.

36 Z. W. Yang, Y. K. Pang, L. M. Zhang, C. X. Lu, J. Chen, T. Zhou, C. Zhang, and Z. L. Wang: ACS Nano 10 (2016) 10912.

37 R. D. P. Wong, J. D. Posner, and V. J. Santos: Sens. Actuators, A 179 (2012) 62.

38 S. J. Park, J. Kim, M. Chu, and M. Khine: Adv. Mater. Technol. 3 (2018) 1700158.

39 E. Puyoo, C. Malhaire, D. Thomas, R. Rafael, M. R'Mili, A. Malchere, L. Roiban, S. Koneti, M. Bugnet, A. Sabac, and M. Le Berre: Appl. Phys. Lett. 110 (2017) 123103.

40 H. S. Shin, J. Y. Ryu, C. Majidi, and Y. L. Park: J. Micromech. Microeng. 26 (2016) 025011.

41 T. D. Nguyen, H. S. Han, H. Y. Shin, C. T. Nguyen, H. Phung, H. Van Hoang, and H. R. Choi: Sens. Actuators, A 266 (2017) 166.

42 K. Sasagawa and J. Narita: Sens. Actuators, A 263 (2017) 610.

43 Z. J. Zhu, R. Y. Li, and T. R. Pan: Adv. Mater. 30 (2018) 1705122.

44 K. Takei, T. Takahashi, J. C. Ho, H. Ko, A. G. Gillies, P. W. Leu, R. S. Fearing, and A. Javey: Nat. Mater. 9 (2010) 821.

45 R. Herbert, J. H. Kim, Y. S. Kim, H. M. Lee, and W. H. Yeo: Materials 11 (2018) 187.

46 J. Park, H. Joshi, H. G. Lee, S. Kiaei, and U. Y. Ogras: ACM Trans. Embedded Comput. Syst. 16 (2017) 156.

47 J. S. Chang, A. F. Facchetti, and R. Reuss: IEEE J. Emerging Sel. Top. Circuits Syst. 7 (2017) 7.

48 C. Mousoulis, X. F. Jiang, N. Raghunathan, and D. Peroulis: IEEE Sens. J. (2017) 927.

49 Y. Khan, M. Garg, Q. Gui, M. Schadt, A. Gaikwad, D. G. Han, N. A. D. Yamamoto, P. Hart, R. Welte, W. Wilson, S. Czarnecki, M. Poliks, Z. P. Jin, K. Ghose, F. Egitto, J. Turner, and A. C. Arias: Adv. Funct. Mater. 26 (2016) 8764.

50 D. Shahrjerdi, S. W. Bedell, A. Khakifirooz, and K. Cheng: Solid State Electron. 117 (2016) 117.

51 P. C. Joshi, T. Kuruganti, and C. E. Duty: Additive Manufacturing: Innovations, Advances, and Applications (CRC Press, Boca Raton, 2016) p. 131.

52 T. Yamashita, S. Takamatsu, H. Okada, T. Itoh, and T. Kobayashi: IEEE Sens. J. 16 (2016) 8840.

53 T. Yamashita, H. Okada, T. Kobayashi, K. Togashi, D. Zymelka, S. Takamatsu, and T. Itoh: IEEE Sens. J 16 (2016) 1.

54 T. Takeshita, T. Yamashita, N. Makimoto, and T. Kobayashi: 2017 Int. Conf. Optical MEMS and Nanophotonics (OMN) (2017) 109.

55 T. Kobayashi, T. Yamashita, N. Makimoto, S. Takamatsu, and T. Itoh: IEEE 29th Int. Conf. Micro Electro Mechanical Systems (MEMS) (2016) 1030.

56 T. Kobayashi, T. Yamashita, K. Togashi, R. Oohigashi, H. Okada, T. Takeshita, S. Takamatsu, and T. Itoh: 19th Int. Conf. Solid-State Sensors, Actuators and Microsystems (Transducers) (2017) 327. 